\title{
Beyond Fighting Fires and Chasing Tails? Chronic Illness Care Plans in Ontario, Canada
}

Grant Russell, MBBS, FRACGP, MFM, $P b D$

Patricia Thille, MA, BSc $(P T)$

William Hogg, MD, CCFP, FCFP

Jacques Lemelin, MD, FCFP

CT Lamont Primary Health Care Research Centre, Elisabeth Bruyère Research Institute, University of Ottawa, Ontario, Canada

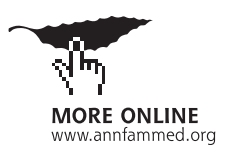

Conflicts of interest: none to report

\section{CORRESPONDING AUTHOR}

Grant Russell, MBBS, PhD

43 Bruyere St

Ottawa, ON, K1N 5C8, Canada

grussell@scohs.on.ca

\begin{abstract}
PURPOSE Recent work has conceptualized new models for the primary care management of patients with chronic illness. This study investigated the experience of family physicians and patients with a chronic illness management initiative that involved the joint formulation of comprehensive individual patient care plans.
\end{abstract}

METHODS A qualitative evaluation, framed by phenomenology, immediately followed a randomized controlled trial examining the effect of external facilitators in enhancing the delivery of chronic condition care planning in primary care. The study, set in Ontario family practices, used semistructured in-depth interviews with a purposive sample of 13 family physicians, 20 patients, and all 3 study facilitators. Analysis used independent transcript review and constant comparative methods.

RESULTS Despite the intervention being grounded in patient-centered principles, family physicians generally viewed chronic illness management from a predominantly biomedical perspective. Only a few enthusiasts viewed systematic care planning as a new approach to managing patients with chronic illness. Most family physicians found the strategy to be difficult to implement within existing organizational and financial constraints. For these participants, care planning conflicted with preexisting concepts of their role and of their patient's abilities to become partners in care. The few patients who noticed the process spoke favorably about their experience.

CONCLUSIONS Although the experiences of the enthusiastic family physicians were encouraging, we found important individual-level barriers to chronic illness management in primary care. These issues seemed to transcend existing organizational and resource constraints.

Ann Fam Med 2008;6:146-153. DOI: 10.1370/afm.793.

\section{INTRODUCTION}

I nternational health care planners are becoming increasingly concerned with the morbidity and mortality associated with chronic illnesses. ${ }^{1,2}$

Although the burden of chronic illness is borne throughout health care systems, it has particular relevance for primary care. ${ }^{1,3}$ Features inherent to primary care-continuity, coordination, and comprehensiveness-are well suited to care of chronic illness, ${ }^{4}$ but many primary care organizations struggle to deliver high-quality care to patients with complex chronic disease (s). ${ }^{5,6}$

Recent research suggests that outcomes may improve if primary care for the chronically ill incorporates enhanced systems for clinical information, evidence-based practice, health system integration, and patient self-management. $^{7-9}$ Many of these dimensions have been incorporated into Wagner and colleagues' Chronic Care Model..$^{10}$ The World Health Organization has extended the model to a national health policy framework. ${ }^{1}$ Mindful of the challenges of implementing such changes across a health care system, some countries, in particular Australia, have oriented primary care chronic disease interventions toward individual practitioners and their patients. ${ }^{11}$ 
Despite the wide promotion of these models, surprisingly little is known about the impact of these strategies on primary care providers or their practices.

This study was designed to evaluate the impact of a holistic, patient-centered, and pragmatic approach to improve the management of chronic disease in the setting of Canadian family practice. The model, called Chronic Illness Care Management (CICM), had 5 essential components (Table 1). Using a structured, written care plan, the CICM was to be delivered to competent patients older than 50 years who had multiple chronic diseases.

The CICM was introduced to family physicians during a series of visits with an experienced outreach facilitator as part of a randomized controlled trial (RCT). ${ }^{12}$ Facilitators were registered nurses with master's degrees in administration or adult education. They visited practices approximately once a month during the course of the study to facilitate practice-organization-level change, first for changes related to prevention and

\section{Table 1. Chronic Illness Care Management (CICM): Model and Care Plan Components}

The CICM was framed as a patient-centered model for primary care management of persons with multiple chronic illnesses. This was to be accomplished through an evaluation of a patient's care requirements via a written care plan prepared collaboratively between a patient and the patient's family physician. Patient health goals and concerns were to be elicited, and 5 components reviewed:

1. Medication review

2. Education and self-care

3. Psychological and social assessment

4. Community integration and social support

5. Prevention

Through this process, patients and physicians could then set mutual goals, with plans for follow-up in planned, scheduled visits.

Physicians were compensated $\$ 300$ for the completion of a care plan. screening practices, then for the CICM phase. The time lines of the study are displayed in Figure 1.

In this article we report findings from a qualitative evaluation nested within the RCT. Our aim was to understand the experience of family physicians and patients regarding the care-planning intervention.

\section{METHODS}

This qualitative study involved in-depth interviews with family doctors, patients, and facilitators in the Ottawa and Hamilton/Wentworth areas of Ontario, Canada. The study used a phenomenological approach to data collection and analysis in an effort to understand the lived experience of study participants. ${ }^{13}$

\section{Physician Participants}

All participating family physicians worked in either Primary Care Networks or Family Health Networks. Both practice types are part of the province's reorganization of primary health care, characterized by patient rosters and blended payment mechanisms with incentives for providing preventive services. ${ }^{14}$ Participating doctors were chosen from family physicians participating in the RCT investigating whether the CICM care plans, implemented with tailored outreach facilitation, improved the quality of life and quality of care of the most complex, chronically ill patients. All physician participants had already participated in a before-andafter study of a facilitator-led intervention designed to enhance the delivery of preventive care. ${ }^{15}$

\section{Recruitment for Interviews}

We sought to gather a purposeful sample of physician participants with varying facilitator-perceived engagement with CICM. In the closing stages of the RCT, the

\section{Figure 1. Study time lines and recruitment.}

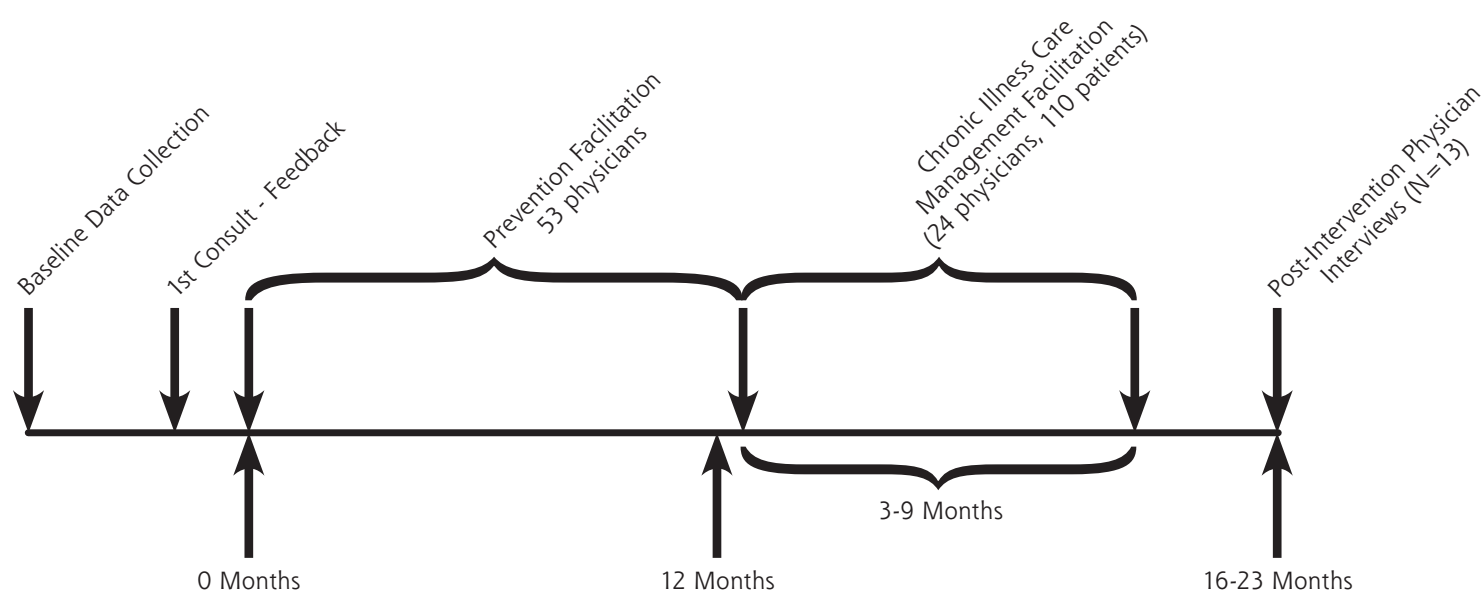


24 participating physicians were invited by facilitators to participate in a qualitative evaluation of the study. Of the 21 who accepted the invitation, a process of purposive sampling was used to identify potential participants. Sampling was primarily based on facilitator perceptions of physician satisfaction and engagement with CICM. Facilitators first identified those physicians who were thought to have embraced the care-planning process. Six participating physicians were perceived to have met this description, 2 with each facilitator. The balance of the sample comprised physicians who were perceived as having neutral or negative views about the process. The 15 physicians approached to participate in the interviews showed maximum variation in terms of sex, practice location (rural or urban), and practice size (solo or group). Thirteen of the 15 physicians who were approached agreed to be interviewed. One refused, citing lack of time. A second consented but was unable to arrange an interview time.

Through prior consent, the research team had access to the names and contact information of patients participating in the RCT. To recruit patients for interviews, consenting physicians were provided a list of their CICM intervention group patients. They identified patients who had completed CICM care planning, whom they thought were able to provide useful insights, and who were capable of participating in an interview in the near future (eg, not currently hospitalized). Identified patients were then telephoned to discern their availability and willingness to participate in an interview. All but 1 of the 21 patients approached agreed. Three chose to be interviewed with a spouse or child present.

All 3 study facilitators were also invited to participate in 2 interviews: 1 at the onset of data collection, the second after early analysis of physician and patient interviews. Physicians and patients were compensated for any participation costs.

\section{Data Collection}

Data collection involved in-depth, face-to-face individual interviews. Physicians were interviewed in their offices, and patients were interviewed in their homes or their physicians' office. Interviews were designed to explore the participant's experience with CICM. Initial interviews followed a written interview guide (available in the online-only Supplemental Appendix at http://

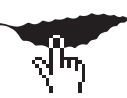

www.annfammed.org/cgi/content/full/6/2/146/

DC1) based on themes identified from a literature review and interviews with the RCT project team. Question sequencing was flexible to allow participant's responses to guide the discussion. The guide was modified progressively in keeping with iterative processes of data collection and analysis, allowing insights from early interviews to inform topics discussed in subsequent interviews.

Interviews with physicians and patients were conducted after the facilitation intervention phase of the RCT, between December 2005 and April 2006. A research associate (P.T.) with a clinical background in physical therapy conducted all but 2 physician and 2 patient interviews, which were conducted by an academic family physician (G.R.). Both interviewed 1 patient together. Patient, physician, and facilitator interviews ranged between 30 and 60 minutes. Interviews continued until the interviewer obtained a clear picture of the participant's experience. Interviews were recorded, transcribed verbatim, and reviewed for accuracy before data analysis. Participants received a short summary of the interview for their review, as well as an accompanying invitation to make comments, corrections, or clarifications.

Textual data provided additional information. A number of documents used as part of the study procedures were reviewed. These documents included the facilitator training manual, minutes of meetings of the study team, and the interim and final reports to the funder. These data provided background and context to understanding the design and implementation experiences of the larger study. Facilitator narratives (written by facilitators following each practice visit), and field notes of the interviewers (written immediately following an interview) were also reviewed in depth (see below).

\section{Data Organization and Analysis}

Immersion-crystallization framed the analysis. ${ }^{16}$ Data organization began with incorporation of the transcripts, field notes, and facilitator narratives data into textual files within NVivo 2.0 (QSR International, Australia). Study documents and facilitator narratives provided context for the interviews and allowed for examination of consistency of physicians' perspectives, whereas the field notes written by interviewers captured immediate impressions of the tone of the interview and provided some insight into interpretation.

Coding was completed by 2 authors (P.T. and G.R.) in 2 stages. First, they read interview transcripts independently to identify key concepts and themes. They then coded transcripts, field notes, and narrative data by a series of major headings (eg, experiences with care planning). These headings had been previously determined from the research questions, interview guides, and existing literature. In the second stage, after a closer review of written node reports on key emergent areas, they identified secondary level codes from the data. Weekly meetings were held to discuss emergent themes, patterns, and connections within and across transcripts. 
The process of analysis was designed to allow us to refute or clarify interpretations through consensus and ongoing reference to the data. Two potential frameworks for understanding the data were discussed at length. The first framework considered the physician participants in terms of whether they had understood the principles of care planning and whether they seemed to have implemented practice change. Reflection and further analyses suggested that the framework described below was best able to represent the data faithfully. Further iterative reflections on coding summaries and on facilitator narratives confirmed these emergent typologies. Theme saturation was reached after the 11 th physician and the 14 th patient interview. The remaining interviews allowed for identifying cases that confirmed and disconfirmed the themes which emerged. The study was approved by the Ottawa Hospital Research Ethics Board.

\section{RESULTS}

The 13 family physician participants varied by sex (11 male, 2 female), clinical experience (from 9 to 35 years in clinical practice), and facilitator-perceived success with implementing CICM (6 were characterized as very successful with CICM). Six worked in rural or semi-urban areas, and 9 worked in solo sites. Seven used electronic medical records, and 8 had nurses or nursepractitioners working in their clinic. Six patients interviewed were male, 14 were female, and they ranged in age from 50 to 90 years. Duration of patient-physician relationships ranged between 1 and 30 years.

The study revealed multiple layers of experiences with care planning among the physician participants. Few participating physicians could articulate with ease the underlying concepts of chronic illness care planning. Individual care planning seemed time consuming and conflicted with many practitioners' perceptions of their role and of their patients' capacities to be partners in care. The patient-centered principles of the intervention seemed inconsistent with many physicians' biomedical models of chronic disease management. The few patients who noticed the process spoke favorably about their experience.

\section{Conceptualizing Care Planning}

Physician participants viewed the CICM approach to planning care as having up to 3 components: systematic chronic illness management; involvement of patients in planning their care; and a broader, more holistic approach to care.

It was clear that most physicians conceptualized the CICM care plan as a systematic, rather than a piecemeal, approach to planning patient care:
I've never done ... care plans for patients. Like you just really do little bits and pieces of it, and it's useful to sort of think in terms of the whole thing ... it's good for the patient because it sort of puts everything together in one place and sort of allows you to see if you're doing what you said you're going to do (Doctor [D] 4).*

Although several welcomed the fact that the care plan "... helped organize my thinking ... it makes you look further and dig a little deeper" (D11), most viewed the process as a framework to ensure the completion of a schedule or series of biomedical clinical tasks. One physician said explicitly: "I think it was more, either myself or the nurse, covering the preventive issues and encouraging them to participate in whatever the preventive measures would be" (D7). Many found it difficult to conceptualize patient problems beyond biomedical disease terms (eg, hypertension, congestive heart failure).

The explicit incorporation of patient needs was not apparent to all physician participants, but several valued the opportunity of being able to integrate the worlds of the patient and the provider.

Probably the biggest difference for me was actually paying a little bit more attention to, in a formal way, to the patient's desires and requests. I think I am, have been, fairly open to what the needs were, but this particular study made me ... stop and look at more than just the medical things, which is what I focused on, mostly. I mean, I'm a rural or country practice, where I know these people and their families quite, quite well; but some of their social needs and other things were in the questionnaire that we had to go over with them, which I probably hadn't addressed as much before, at least not formally (D1).

Several physicians viewed the care-planning process as opening the door to a more comprehensive and holistic care:

Even though it's not a pill I'm giving her, it's something that's making her healthier because, you know, it's a, it's a change in her social condition, so ... she got subsidized better accommodation, she got more money to buy food and will get some subsidies for her orthotics and shoes" (D4).

\section{The Enthusiasts}

It was clear that some of the participating physicians embraced CICM. Facilitators described these participants as being open to the model from the outset, positive about its implementation, and able to provide constructive solutions for the future. They came from varied practices and both rural and urban communities. These enthusiasts described core concepts of CICM with ease and provided rich descriptions of their process and experiences of using the care plan tool as an adjunct to enhancing chronic illness management.

\footnotetext{
* All quotations have been edited slightly to improve readability.
} 
Enthusiasts acknowledged the contribution of the facilitators in highlighting a need for a change. Speaking of the facilitator's impact, one older physician suggested that "what she did is actually turn my whole thinking around ... (from) acute episodic to preventative" (D11). Enthusiasts' views of their roles changed in a positive way. One male physician who worked in a solo site of a group practice remarked, "In some ways (it was) more satisfying to me anyway, like I'm more of a manager than sort of just putting out fires. I mean I keep putting out fires and they keep starting" (D4).

The process caused them to reflect upon the deficiencies of their usual model of care:

I'm not actually doing anything proactive to try and help them out, but you're doing reactive, reactive, reactive, and ... always chasing your tail. Um, the thing I liked about this was it was more proactive. (I was able to ask) "What can we do to keep you out of trouble?" (D12).

They spoke of surprising new insights into patients with whom they had long standing relationships:

I tend to assume things about patients ... [that] I know everything about them that I need to know medically, and that's not true. You've got to be careful about that and that's very humbling (D13).

One rural family physician related a patient's response when he asked her to describe her biggest health challenge:

I thought, her biggest challenge was all sorts of pain issues ... she's diabetic, has terrible rheumatoid arthritis,... (but) she says, "No it's being able to get my groceries and cook my dinners and stay in my house. That's my biggest challenge

I don't care about the pain, all those other issues, I mean, they're only concerning as far as they affect my function." ... She was basically able to say, "all those other things only matter in so far as helping me stay in my house ... that's my only goal."

And so for me that was ... an eye opener. It was, Okay it's not about me dealing with your symptoms, it's me dealing with your end goal, and so it became less focused on medications, more focused on getting her a scooter, so she could scooter up to the grocery store-for 4 or 5 months of the year she is totally independent.... I hadn't thought of that. My approach was no longer, you know, symptom, treatment; symptom, treatment; symptom, treatment. When she starting complaining about these things,... (I now think) "Okay, what do I do for this so that it doesn't get out of control and stop her from being at home?" (D12).

\section{The Unenthused}

In contrast, many physician participants were either dismissive of the CICM approach to care planning or overwhelmed by its demands. They spoke at length about the challenges and barriers to planning care in general and about using the CICM care plan format more specifically. Their descriptions of the process of care planning with patients were cursory and lacking in color.

'This is Not How Doctors and Patients Work Together' Several physicians told us unequivocally that what CICM asked of them was "not my role": "I think somebody else could have done it just as well... It's not my training ... it was interesting, but I think obviously somebody else could have done better" (D6); and "... typically a nurse would be able to go through it. I don't think that a family physician would be able to fill one of those [care plans] out" (D5).

Some noted that the patient's role of involvement in the care plan was unrealistic, believing that their patients were not capable of engaging in the sorts of decisions essential to collaborative care planning: "I don't think those are the highly sophisticated bunch of folk.... None of them ... really could participate in that shared model of care" (D2).

'This is Not Different From What I Already Do' Planning and scheduling care were pivotal to the care plan. Although the principle of scheduling care was not challenged, some participants remarked they already provided planned follow-up with these patients, sometimes obliquely through the expiration of prescriptions for long-term medications. When asked about his perception of CICM's proactive model of care, a more openly resistant physician told us:

Family physicians like to think that we do this all the time. We don't, perhaps, sit down in an hour, and decide, you know, "We're going to look at your chest pain, we could look at psychosocial issues." Sometimes we do it if we've got time .... (D2).

Many of the resistant physicians seemed more comfortable with the explicitly biomedical components of the care plan (medication reviews and prevention activities), and gave less priority to psychological and social issues.

Even though several enthusiasts modified the tool and discovered new insights into their long-term patients, the unenthusiastic physicians found the tool to be inflexible and, at times, superfluous to their needs:

It was kind of unusual because a lot of these people I've known for a long, long time ... a lot of it's written down on in the charts, so it was sort of, it felt a little redundant to go through this with them. Kind of awkward, actually ... (D9).

'Our Biggest Problem Was Resource Constraints' For many physician participants the CICM was either financially or organizationally impractical. Notwithstanding the reimbursement associated with completion 
of care plans, it was difficult for many to schedule 30 minutes for the first CICM planning visit. Many physicians saw allied health professionals, both within and outside family practices, as necessary to making CICM work in the future: "Solo GP doctors, I don't think, can manage this without those supports" (D7). A number remarked on what they believed to be a lack of community-based resources to complement patient care.

\section{Patients' Perspectives}

Whereas most patients barely noticed any change with CICM, some recalled experiencing a different approach to care with their physician, especially during the initial planning visits. Of those noting a difference in their care, all but 1 was paired with an 'enthusiastic' physician.

One gentleman with osteoporosis described his first structured visit:

It was really impressive.... It wasn't just a quick little visit amidst a busy day. It was something different. It was something very dedicated, very planned. And it was nice to be able to talk on something which, perhaps, I was on the other side looking in. You know, he wasn't looking at my leg or my arm, or an illness" (Patient [P] 18).

Those reporting a positive impact believed the care-planning process gave an opportunity for the physician to know them more as a person:

I remember her asking, um, you know, what do I do if I get upset? You know, do I have someone to talk to? ... just things like that... I think that's a good idea, because a lot of people won't approach their doctors about personal things (P8).

Some believed that their care coordination had improved as a result of care planning and reported perceived personal benefits, such as taking fewer medications, reduced anxiety about their conditions, having new strategies to manage their health problems, or functional improvements. Very few patients, however, articulated improved community linkages or enhanced self-management skills. Even though all patients welcomed the additional time available through CICM care planning, a number speculated as to whether it was cost- or time-effective for physicians.

\section{DISCUSSION}

Initiatives to improve the care of chronically ill patients have varied in philosophy and design. ${ }^{17}$ Recent US models have emphasized broad systematic change, often delivered within the relatively controlled environment of managed care organizations. ${ }^{18}$ By contrast, Australia has implemented a pragmatic strategy, part of which involves financial incentives for family doctors completing written patient care plans. ${ }^{19}$ Our study provided an opportunity to understand the experiences of patients and physicians with an intervention similar to the Australian model. The findings provide insight into some unique challenges associated with primary care management of chronic illness.

At the heart of the practitioner experiences was a contrast between the enthusiasts and those practitioners who seemed unmoved by the initiative. As described, the enthusiasts were evenly spread among facilitators, community locations, and practice characteristics. Their willingness to embrace ongoing learning and quality improvement seems consistent with early adopters of change. ${ }^{20}$ For the enthusiasts, CICM style care planning moved traditional family practice beyond reactive care ("chasing tails" and "fighting fires") to a more proactive and comprehensive model. Important lessons follow from the experiences of the unenthusiastic, however.

\section{Barriers to Change}

The successful implementation of change in clinical care depends on multiple factors, including features of the change itself, the level and nature of the evidence, the context or environment into which the research is to be placed, and the method of facilitation. ${ }^{20,21}$ Participating physicians generally viewed the CICM initiative as time intensive and unrealistic for widespread implementation. All physician participants had an enduring relationship with an experienced facilitator, and they experienced implementing change in practice with regard to preventive care that led to statistically significant improvements. ${ }^{15}$ They worked in practices with similar supports (human resource and technology) and in the same communities. Even so, there were distinct differences among physicians in levels of enthusiasm for a comprehensive, patient-centered, planned care approach. The enthusiastic physicians spoke of the promise of patient-centered care planning and of small ways in which they have already integrated components of the care plan. By contrast, the skepticism of the unenthusiastic was reinforced by difficulties in accessing allied health professionals, limited availability of community resources, and lack of a supportive fee schedule. Although other studies have listed or identified some of the same barriers, 5,19,22 this study illuminates what we believe to be fundamental barriers at the physician level that may need to be considered in future attempts in improving chronic illness management in primary care.

Most physicians welcomed the concept of more patient involvement in care, but the idea of collaborating with patients to develop a comprehensive plan focusing on shared goals conflicted with some of the unenthusiastic physicians' self-perceived job responsibilities. The 
concept that shared and proactive care is "not my job" would seem to be an important barrier to the success of collaborative models emphasizing the value of patientphysician partnership..$^{8,23}$ Similarly, a number of the unenthusiastic participants doubted that their chronically ill patients could manage to fulfill a role as their own principal caregiver. ${ }^{8}$ Not surprisingly, few acknowledged their own role in preparing patients for such a change.

Others have reported how primary care providers are often convinced that they provide optimal chronic illness care. ${ }^{24}$ As in Australia, our lack of a tool for auditing patient-centered, collaborative chronic illness care made it difficult for the physicians to measure the quality of their chronic illness care. Heightening awareness through the use of either administrative data or validated tools, such as the Assessment of Chronic Illness Care ${ }^{25}$ or the Patient Assessment of Chronic Illness Care, ${ }^{26}$ could highlight gaps in care and potentially increase physicians' motivation to change.

\section{Future Implications}

Although the facilitators seemed to work well with the more reluctant family physicians, the lack of organizational support and the complexities of unenthusiastic physician perspectives stood in the way of meaningful change. Despite the evidence that the initiative had tapped a need in the enthusiasts, pervasive individual barriers combined with a lack of system based support suggests that this approach is unlikely to have a major impact in the Ontario health care system at this time. After more than 3 years of experience with a similar care-planning strategy in Australia, Wilkinson et al found that $10 \%$ of the general practice workforce was responsible for completing $80 \%$ of all care plans. ${ }^{19}$ Our findings point to several practical strategies to consider in chronic illness management in primary care.

The partnership role and responsibility changes in collaborative chronic illness care models may require a range of strategies at an individual level to help the different groups implement effective chronic care management. Some argue medical education does not prepare physicians for the demands of a complex, collaborative health care environment. ${ }^{8}$ With wide policy interest in primary care delivery of chronic illness care, it may be time for professional organizations to reconsider whether undergraduate or postgraduate training programs should be reorientated toward the demands of a collaborative health care environment where patients are understood as their own primary caregiver.

Other studies have spoken of the importance of group culture ${ }^{27}$ and colleague support ${ }^{22}$ in addressing some of individual barriers to chronic care management. Opinion leaders and cross-practice mentorship or collaboration may be helpful to foster the shift to patient-centered and proactive chronic illness management. Our findings suggest that facilitation needs to take account of the physicians' approach to care and at the very least assist with practice audit techniques to help practices identify gaps in the quality of clinical care, particularly those around patient-centered, collaboratively oriented care.

Transferability of the study findings is limited in that physician participants all practiced in relatively small capitated practices in 2 regions of Ontario. Each had already participated in 2 distinct intervention studies designed to investigate new methods of delivering primary care. All had at least 1 decade of postgraduation experience. Although the sampling technique included an awareness of the need to search for alternative and disconfirming cases, we may have been unable to capture different perspectives shared by other practitioners. Specifically, more recent medical graduates or physicians working in interdisciplinary primary care settings may have been more supportive of the principles of collaborative chronic condition care. Our methodology asked physicians to nominate patients for interview, thereby possibly excluding patients with negative experiences of their care.

Our use of phenomenology was well suited for capturing patient and practitioner experience. Although we gained a good understanding of practitioner orientations to chronic condition management, other methods, particularly those using ethnographic techniques of direct observation, are better suited for understanding behavior in the clinical setting. Epidemiologic methods would be required to examine the influence of practitioner orientation on adherence to clinical guidelines or effectiveness of care.

Implementing comprehensive, patient-centered chronic illness care management involves more than organizational change. Our study highlights the importance of the personal attributes and perspectives of individuals in addition to larger system issues. More complex barriers to change, including attitudes and professional culture, should be considered in future attempts to improve the delivery of chronic illness care in primary care practices. Our findings illuminate the need for additional methods of support for both family physicians and patients while they transition to the adjusted roles and responsibilities of collaborative and proactive management of chronic illness.

To read or post commentaries in response to this article, see it online at http://www.annfammed.org/cgi/content/full/6/2/146.

Key words: Chronic disease; patient care management; primary health care; family practice; attitude of health personnel; patient care planning; patient-centered care; patient participation 
Submitted November 13, 2006; submitted, revised, August 30, 2007; accepted September 17, 2007.

A version of this report was presented at the Australian General Practice and Primary Health Care Research Conference (poster), Perth, Australia, July 2006; and at the North America Primary Care Research Group, Tucson, Arizona, October 16, 2006.

Funding support: Funding for this research was provided by the Ontario Ministry of Health and Long-Term Care Primary Health Care Transition Fund.

Disclaimer: The views expressed in this report are the views of the authors and do not necessarily reflect those of the Ontario Ministry of Health and Long-Term Care.

Acknowledgments: Robert Geneau, PhD, assisted with the design of the qualitative evaluation.

\section{References}

1. World Health Organization. Innovative Care for Chronic Conditions: Building Blocks for Action. Global Report. Geneva: Noncommunicable Diseases and Mental Health, World Health Organization; 2002.

2. World Health Organization. Observatory on health care for chronic conditions. Chronic conditions: the global burden. 2006. http:// www.who.int/chronic_conditions/burden/en/index.html. Accessed Aug. 10, 2006

3. Fortin M, Bravo G, Hudon C, Vanasse A, Lapointe L. Prevalence of multimorbidity among adults seen in family practice. Ann Fam Med. 2005;3(3):223-228

4. Rothman AA, Wagner EH. Chronic illness management: what is the role of primary care? Ann Intern Med. 2003;138(3):256-261.

5. Hroscikoski MC, Solberg LI, Sperl-Hillen JM, Harper PG, McGrail MP, Crabtree BF. Challenges of change: a qualitative study of chronic care model implementation. Ann Fam Med. 2006;4(4):317-326.

6. Epping-Jordan JE, Pruitt SD, Bengoa R, Wagner EH. Improving the quality of health care for chronic conditions. Qual Safe Health Care. 2004;13(4):299-305.

7. Wagner EH, Austin BT, Davis C, Hindmarsh M, Schaefer J, Bonomi A. Improving chronic illness care: translating evidence into action. Health Aff (Millwood). 2001;20(6):64-78.

8. Holman $\mathrm{H}$. Chronic disease-the need for a new clinical education. JAMA. 2004;292(9):1057-1059.

9. Tsai AC, Morton SC, Mangione CM, Keeler EB. A meta-analysis of interventions to improve care for chronic illnesses. Am J Manag Care. 2005;11(8):478-488.

10. Bodenheimer T, Wagner EH, Grumbach K. Improving primary care for patients with chronic illness: the chronic care model, Part 2. JAMA. 2002;288(15):1909-1914.

11. Department of Health and Ageing. Chronic disease management (CDM) Medicare items (new from July 1, 2005). Australian Government. 2006 June 29. http://www. health.gov.au/internet/wcms/publishing.nsf/Content/pcd-programs-epc-chronicdisease.
12. Hogg W, Lemelin J, Russell G. A randomised controlled trial of care planning in family practice: interim results. Family Medicine Forum 2005. Vancouver, British Columbia.

13. Patton MQ. Qualitative Research and Evaluation Methods. 3rd ed. Thousand Oaks, CA: Sage Publications; 2002.

14. Muldoon L, Rowan M, Geneau R, Hogg W, Coulson D. Models of primary care service delivery in Ontario: why such diversity? Healthc Manage Forum. 2006;19(4):18-23.

15. Hogg W, Lemelin J, Soto E, Moroz I. Sustainability of Improved Preventive Services' Delivery Resulting from Outreach Facilitation (poster). NAPCRG Annual Meeting. October 16, 2006. Tucson, AZ.

16. Borkan J. Immersion/crystallization. In: Crabtree BF, Miller WL, editors. Doing Qualitative Research. 2nd ed. Thousand Oaks, CA: Sage Publications; 1999:179-194.

17. Ouwens $M$, Wollersheim $H$, Hermens $R$, et al. Integrated care programmes for chronically ill patients: a review of systematic reviews. Int J Qual Health Care. 2005;17(2):141-146.

18. Bodenheimer T, Wagner EH, Grumbach K. Improving primary care for patients with chronic illness. JAMA. 2002;288(14):1775-1779.

19. Wilkinson D, Ott K, Morey S, et al. Evaluation of the Enhanced Primary Care (EPC) Medicare Benefits Schedule (MBS) items and the General Practice Education, Support and Community Linkages Program (GPESCL). Department of Health and Ageing, Australian Government Department of Health and Ageing; 2003. http://www. health.gov.au/epc/epcevaluation.htm.

20. Kitson A, Harvey G, McCormack B. Enabling the implementation of evidence based practice: a conceptual framework. Qual Health Care. 1998;7(3):149-158.

21. Grol R, Grimshaw J. From best evidence to best practice: effective implementation of change in patients' care. Lancet. 2003;362(9391): $1225-1230$

22. Oldroyd J, Proudfoot J, Infante FA, et al. Providing healthcare for people with chronic illness: the views of Australian GPs. Med J Aust. 2003;179(1):30-33

23. Bodenheimer T, Lorig K, Holman H, Grumbach K. Patient self-management of chronic disease in primary care. JAMA 2002;288(19):2469-2475.

24. Wagner EH, Glasgow RE, Davis C, et al. Quality improvement in chronic illness care: a collaborative approach. Jt Comm J Qual Improv. 2001;27(2):63-80.

25. Bonomi AE, Wagner EH, Glasgow RE, VonKorff M. Assessment of chronic illness care $(\mathrm{ACIC})$ : a practical tool to measure quality improvement. Health Serv Res. 2002;37(3):791-820.

26. Glasgow RE, Wagner EH, Schaefer J, Mahoney LD, Reid RJ, Greene SM. Development and validation of the Patient Assessment of Chronic Illness Care (PACIC). Med Care. 2005;43(5):436-444.

27. Rundall TG, Shortell SM, Wang MC, et al. As good as it gets? Chronic care management in nine leading US physician organisations. BMJ. 2002;325(7370):958-961. 